\title{
ON BESSEL FUNCTIONS AND RATE OF CONVERGENCE OF ZEROS OF LOMMEL POLYNOMIALS
}

\author{
P. FEINSILVER AND R. SCHOTT
}

\begin{abstract}
In this note, we solve an open problem of Flajolet and Schott concerning the rate of convergence of the zeros of Lommel polynomials to the zeros of a Bessel function (considered as a function of the order). The average case analysis of dynamic data structures was the initial motivation for this investigation. The Maple program, whose results are reported at the end, illustrates that our result is quite good.
\end{abstract}

\section{INTRODUCTION}

Coulomb [1] first investigated the zeros of the Bessel functions $J_{\nu}(x)$, with $x$ fixed. He observed that the zeros converge to the negative integers as $\nu \rightarrow-\infty$. More recently, Flajolet and Schott [2] gave a quantitative version of Coulomb's result and solved some combinatorial problems related to nonoverlapping partitions. They mentioned that the dynamic analysis of data structures apparently required investigating the speed of convergence of zeros of Lommel polynomials to zeros of Bessel functions. This provided the initial motivation for this investigation. It is to be noted that Lommel polynomials arise via the convergents of the continued fraction expansion converging to the Bessel function. It is likely that our technique may prove useful in similar cases for other classes of functions.

\section{LOMMEL POLYNOMIALS AND BESSEL FUNCTIONS}

The reader is referred to Watson's treatise [3] for the basic theory of Bessel functions. Let us just recall that the Bessel function $J_{\nu}$ is defined by

$$
J_{\nu}(z)=\left(\frac{z}{2}\right)^{\nu} \sum_{n=0}^{\infty} \frac{(-1)^{n}(z / 2)^{2 n}}{n ! \Gamma(n+\nu+1)}
$$

and satisfies the fundamental recurrence

$$
2 \nu x^{-1} J_{\nu}(x)=J_{\nu+1}(x)+J_{\nu-1}(x) .
$$

In the following, the argument of the Bessel function is fixed and $\nu$ is variable,

Received by the editor June 11, 1991.

1991 Mathematics Subject Classification. Primary 41A25; Secondary 33C10. 
and will be denoted by $x$. Consider the (modified) Lommel polynomials

$$
H_{m}(x)=\sum_{n=0}^{[m / 2]}\left(\begin{array}{c}
m-n \\
n
\end{array}\right)(-1)^{n}(x+n)_{m-2 n}
$$

with $(x)_{a}=x(x+1) \cdots(x+a-1)$.

Note. Throughout the following, $x$ is any complex number that is not an integer.

We have, in the notation of $[3$, p. 294ff]

$$
H_{m}(x) \equiv R_{m, x}(2) \text {. }
$$

We let

$$
J(x) \equiv J_{x}(2)=\sum_{n=0}^{\infty} \frac{(-1)^{n}}{n ! \Gamma(x+1+n)} .
$$

For $m \rightarrow \infty$, recalling that $x$ is nonintegral,

$$
J(x+m)=\frac{1}{\Gamma(x+m+1)}\left[1+O\left(\frac{1}{m}\right)\right]
$$

and

$$
J(-x-m)=\frac{1}{\Gamma(-x-m+1)}\left[1+O\left(\frac{1}{m}\right)\right],
$$

where the $O$-terms are uniform (in $x$ ) on compact sets. The principal formula required is the following, which may be found in [3, p. 295]:

$$
H_{m}(x)=\frac{\pi}{\sin \pi x}\left[J(x+m) J(1-x)+(-1)^{m} J(-x-m) J(x-1)\right] .
$$

Now let $\mu$ be a positive integer. Define the domain $\Omega_{\mu}=\{x: 0<|x+\mu|<$ 1\}. The first lemma is related to Hurwitz' result for Lommel polynomials [3, p. 302].

Lemma 1. On $\Omega_{\mu}, H_{m}(x) / \Gamma(x+m)$ as $m \rightarrow \infty$ converges to $J(x-1)$ uniformly on compact subsets ('normally').

Proof. By $(*)$,

$$
\begin{aligned}
\frac{H_{m}(x)}{\Gamma(x+m)}= & O\left(\frac{1}{\Gamma(x+m) \Gamma(x+m+1)}\right) \\
& +\frac{\pi}{\sin \pi x} \frac{(-1)^{m} J(-x-m) J(x-1)}{\Gamma(x+m)} .
\end{aligned}
$$

The first term is negligible. We have to show that the coefficient of $J(x-1)$ in the second term converges normally to 1 . In fact,

$$
\begin{aligned}
\frac{\pi}{\sin \pi x} \frac{(-1)^{m} J(-x-m)}{\Gamma(x+m)}= & \frac{\pi}{\sin \pi(x+\mu)} \frac{(-1)^{m+\mu}}{\Gamma(x+\mu) \Gamma(1-x-\mu)} \\
& \times \frac{\Gamma(x+\mu)}{\Gamma(x+m)} \frac{\Gamma(1-x-\mu)}{\Gamma(1-x-m)}\left[1+O\left(\frac{1}{m}\right)\right],
\end{aligned}
$$


where we note that the $O$-terms are uniform on compact subsets of $\Omega_{\mu}$. By the reflection formula for the gamma function, the first two factors reduce, and thus the expression is asymptotic to

$$
(-1)^{m+\mu} \frac{\Gamma(x+\mu) \Gamma(1-x-\mu)}{\Gamma(x+m) \Gamma(1-x-m)}=1 .
$$

Recall that the zeros of $J(x)$ are very close to negative integers as $x \rightarrow-\infty$ [1].

Lemma 2. Let $J(\zeta-1)=0$. Let $\zeta_{m}$ be the zero of $H_{m}(x)$ closest to $\zeta$. Then, as $m \rightarrow \infty$, we have the asymptotic relation

$$
\left|\zeta_{m}-\zeta\right| \sim\left|J^{\prime}(\zeta-1)\right|^{-1}\left|\frac{H_{m}(\zeta)}{\Gamma(\zeta+m)}\right|
$$

Proof. (The idea of the proof is similar to that of Newton's method for solving equations.) Let $f_{m}(x)=H_{m}(x) / \Gamma(x+m)$. So $f_{m}$ and $H_{m}$ have the same roots near $\zeta$. By the mean value theorem, there exists $\xi_{m}$, e.g., on the segment between $\zeta$ and $\zeta_{m}$, such that

$$
f_{m}(\zeta)-f_{m}\left(\zeta_{m}\right)=\left(\zeta-\zeta_{m}\right) f_{m}^{\prime}\left(\xi_{m}\right) .
$$

Thus, since $f_{m}\left(\zeta_{m}\right)=0$, as $m \rightarrow \infty$,

$$
\left|\zeta-\zeta_{m}\right|=\left|\frac{f_{m}(\zeta)}{f_{m}^{\prime}\left(\xi_{m}\right)}\right| \sim\left|f_{m}^{\prime}(\zeta)\right|^{-1}\left|f_{m}(\zeta)\right|,
$$

where, since $\left\{f_{m}(x)\right\}$ converges normally to $J(x-1)$ (by Cauchy's formula), the derivatives are equicontinuous. For large $m$, we can replace $f_{m}^{\prime}(\zeta)$ by $J^{\prime}(\zeta-1)$.

Finally we have:

Theorem. Let $\zeta$ denote a zero of $J(x-1)$. Let $\zeta_{m}$ denote the zero of $H_{m}(x)$ nearest to $\zeta$. Then, as $m \rightarrow \infty$,

$$
\left|\zeta_{m}-\zeta\right| \sim\left|\frac{\pi}{\sin \pi \zeta} \frac{J(1-\zeta)}{J^{\prime}(\zeta-1)} \frac{1}{\Gamma(\zeta+m) \Gamma(\zeta+m+1)}\right| .
$$

Proof. We apply $(*)$ at $x=\zeta$, so that the $J(x-1)$ term vanishes. Thus,

$$
\frac{H_{m}(\zeta)}{\Gamma(\zeta+m)}=\frac{\pi}{\sin \pi \zeta} J(1-\zeta) \frac{J(\zeta+m)}{\Gamma(\zeta+m)} .
$$

Combining (3) with Lemma 2 yields the result.

Remark. Relation (10) may be written in the form

$$
\left|\zeta_{m}-\zeta\right| \sim\left|\frac{J(1-\zeta)}{J^{\prime}(\zeta-1)} \frac{\Gamma(-\zeta-m)}{\Gamma(\zeta+m)}\right|
$$

via the reflection formula for the gamma function.

Some numerical results illustrating the theorem are given in Table 1. 
TABLE 1

\begin{tabular}{|c|c|c|c|c|c|c|}
\hline & \multicolumn{2}{|c|}{$\left|\xi^{L}-\xi_{1}\right|$} & \multicolumn{2}{c|}{$\left|\xi^{L}-\xi_{5}\right|$} & \multicolumn{2}{c|}{$\left|\xi^{L}-\xi_{8}\right|$} \\
\hline$m$ & Theory & Practice & Theory & Practice & Theory & Practice \\
\hline 4 & $1 \times 10^{-3}$ & $9 \times 10^{-4}$ & & & & \\
\hline 5 & $1 \times 10^{-5}$ & $4 \times 10^{-5}$ & & & & \\
\hline 6 & $1 \times 10^{-6}$ & $1 \times 10^{-6}$ & & & & \\
\hline 7 & $3 \times 10^{-8}$ & $2 \times 10^{-8}$ & $8 \times 10^{-2}$ & $4 \times 10^{-2}$ & & \\
\hline 8 & $4 \times 10^{-10}$ & $4 \times 10^{-10}$ & $7 \times 10^{-3}$ & $4 \times 10^{-3}$ & & \\
\hline 9 & & & $3 \times 10^{-4}$ & $2 \times 10^{-4}$ & & \\
\hline 10 & & & $1 \times 10^{-5}$ & $8 \times 10^{-6}$ & $8 \times 10^{-2}$ & $4 \times 10^{-2}$ \\
\hline 11 & & & $3 \times 10^{-7}$ & $2 \times 10^{-7}$ & $7 \times 10^{-3}$ & $4 \times 10^{-3}$ \\
\hline 12 & & & $5 \times 10^{-9}$ & $4 \times 10^{-9}$ & $3 \times 10^{-4}$ & $2 \times 10^{-4}$ \\
\hline 13 & & & & & $1 \times 10^{-5}$ & $8 \times 10^{-6}$ \\
\hline 14 & & & & & $3 \times 10^{-7}$ & $2 \times 10^{-7}$ \\
\hline 15 & & & & & $5 \times 10^{-9}$ & \\
\hline
\end{tabular}

$\xi_{i}$ denotes a zero of $J(\nu)=J_{\nu}(2)$,

$\xi^{L}$ denotes a zero of a Lommel polynomial,

We have (approximately) $\xi_{1}=0.2538058170, \xi_{5}=-4.9997743198$,

$\xi_{8}=-7.9999999961$.

\section{ACKNOWLEDGMENTS}

This work has been supported in part by the P.R.C. Mathématiques et Informatique and by NATO grant \#86/0321.

\section{BIBLIOGRAPHY}

1. J. Coulomb, Sur les zéros des fonctions de Bessel considérées comme fonction de l'ordre, Bull. Sci. Math. 60 (1936), 297-302.

2. P. Flajolet and R. Schott, Non-overlapping partitions, continued fractions, Bessel functions, and a divergent series, European J. Combin. 11 (1990), 421-432.

3. G. N. Watson, A treatise on the theory of Bessel functions, Cambridge Univ. Press, 1980.

Department of Mathematics, Southern Illinois University, Carbondale, Illinois 62901

E-mail address: GA3613@SIUCVMB.bitnet

Centre de Recherche en INFormatique de Nancy, INRIA-LORRAINE, Université de NANCY 1, B.P. 239, 54506-VANDOEUVRE-LĖS-NANCY, FranCE

E-mail address: schott@loria.crin.fr 